\title{
Renal Trauma: The Rugby Factor
}

\author{
Catherine M. Freeman ${ }^{\mathrm{a}}$ Michael E. Kelly ${ }^{\mathrm{a}} \quad$ Gregory J. Nason ${ }^{\mathrm{a}} \quad$ Barry B. McGuire \\ Aoife Kilcoyne $^{\mathrm{b}} \quad$ John Ryan ${ }^{c} \quad$ Gerald Lennon $^{\mathrm{a}}$ David Galvin ${ }^{\mathrm{a}}$ \\ David Quinlan ${ }^{\mathrm{a}} \quad$ David Mulvin $^{\mathrm{a}}$ \\ aDepartment of Urology; 'Department of Radiology; and 'Emergency Department, St Vincent's University Hospital, Elm Park, \\ Dublin, Ireland
}

\section{Key Words}

Abdominal trauma • Sports trauma • Renal trauma •

Trauma management

\section{Abstract}

Introduction: Renal trauma accounts for $5 \%$ of all trauma cases. Rare mechanisms of injuries including sports participation are increasingly common. Rugby-related trauma poses a conundrum for physicians and players due to the absence of clear guidelines and a paucity of evidence. Our series highlights traumatic rugby-related renal injuries in our institution, and emphasize the need for international guidelines on management. Methods: A retrospective review of all abdominal traumas between January 2006 and April 2013, specifically assessing for renal related trauma that were secondary to rugby injuries was performed. All patients' demographics, computerized tomography results, hematological and biochemical results and subsequent management were recorded. Results: Five male patients presented with rugby-related injuries. Mean age was 21 years old. All patients were hemodynamically stable and managed conservatively in acute setting. One patient was detected to have an unknown pre-existing atrophic kidney that had been subsequently injured, and was booked for an elective nephrectomy an 8-week interval. Conclusion: Rugby-related trauma has generated essential attention. This paper serves to highlight this type of injury and the need for defined guidelines on role of imaging and international consensus on timing of return to contact sport, in both professional and amateur settings.

Copyright $\odot 2014$ S. Karger AG, Basel

\section{KARGER}

Fax +4161306 1234

E-Mail karger@karger.ch

www.karger.com
(C) 2014 S. Karger AG, Basel

1015-9770/14/0083-0133\$38.00/0

Accessible online at:

www.karger.com/cur

\section{Introduction}

Renal trauma accounts for 5\% of all trauma cases [1]. Blunt trauma due to road traffic accidents, falls and assaults are the major contributing factors [2]. However, albeit a rarer mechanism of injury, sports participation are an increasingly common presentation. Pediatric data from North American studies have observed that 16-30\% of renal trauma is secondary to sporting activity [3].

Rugby-related trauma has had significant media attention with concerns over player safety. Governing bodies have introduced new measures including the on-pitch 'concussion bin' in an effort to improve the safety of the sport. Renal specific trauma poses a conundrum for treating physicians and team medics in respect to the timing of returning to contact training, which is especially important in the professional era.

Traditionally, surgical management was the mainstay treatment of renal trauma. However, in recent years a conservative approach has been advocated [4]. The role and timing of follow-up imaging also remains a contentious issue [5]. Evidence supports no interval imaging for Grade I and II renal trauma [6, 7]. The European Association of Urology (EAU) has published a management algorithm for traumatic renal injuries [7] (fig. 1), however only half of urologists in Great Britain utilize them [8].

This paper serves to highlight traumatic rugby-related renal injuries and the need for international guidelines on role of imaging and when is it safe to return to contact sport. 


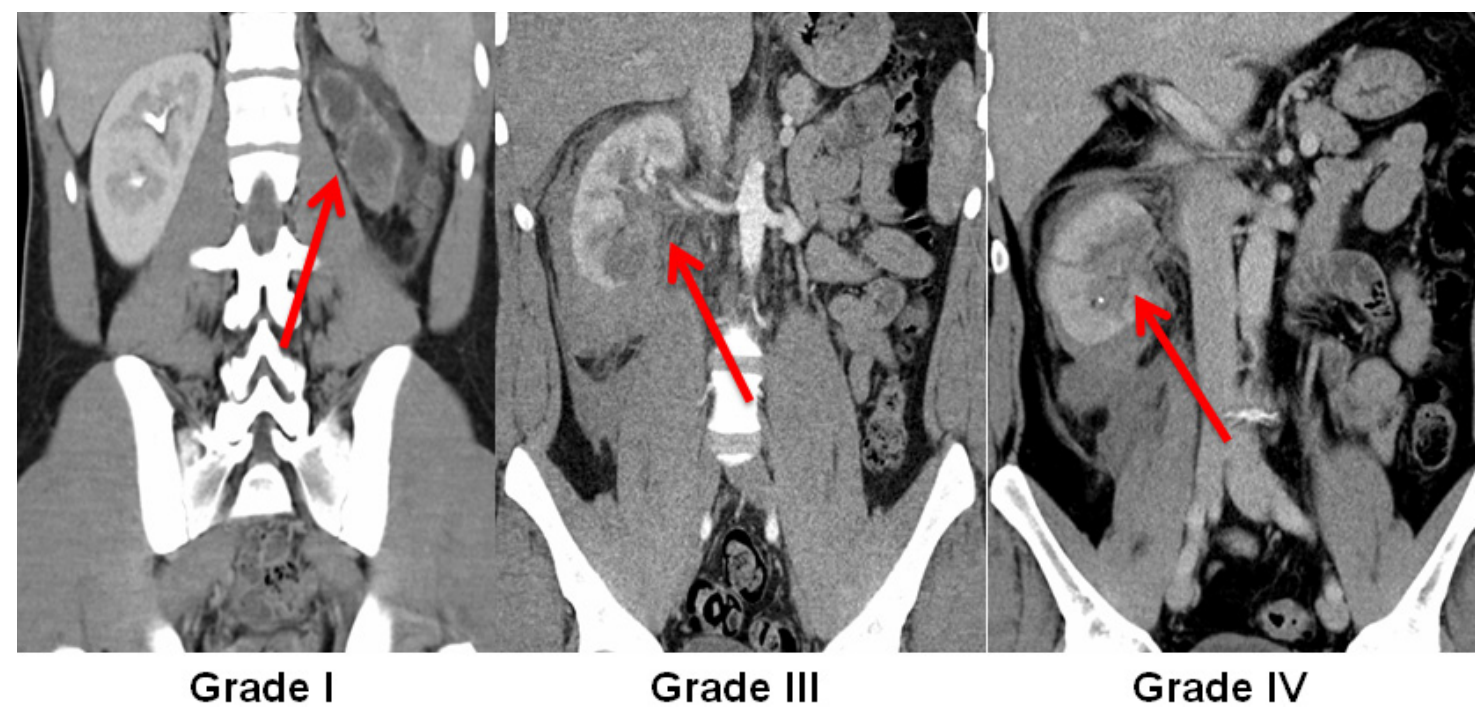

Fig. 1. Coronal images on the 3 grades on renal injury in this series (according to AAST-OIS).

\section{Methods}

A retrospective review was conducted in a tertiary referral university hospital emergency department (ED), which assesses over 45,000 patients per year. The ED serves a population catchment exceeding 350,000 people. Between January 2006 and April 2013, a retrospective medical record analysis was conducted of all patients (aged $>16$ years) that were reviewed with abdominal trauma in the ED. We specifically examined for renal related trauma that were secondary to rugby injuries (organized rugby including semi and professional players). All patients had a triphasic computed tomography (CT) scan (with non-contrast, contrast and delayed phases) of the abdomen at time of presentation (as per departmental protocol) to assess and define the renal injury and/or any associated injuries. Patient demographics, presenting features, timing and position of injury, imaging and subsequent American Association for the Surgery of Trauma Organ Injury Scale (AAST-OIS) grading was recorded. A consultant radiologist reported all images. All patients were arranged to be followed-up in the urology outpatients department prior to recommencing contact training.

\section{Results}

During the study period, 5 male patients presented with rugby-related injury. All patients had no significant medical or surgical history. However, patient five on imaging was detected to have an unknown pre-existing atrophic left kidney, which had been subsequently injured. The mean age of admitted patients was 21 years old (range 19-26 years). The average weight was 101.2 kg (range 80-118 kg).
Sixty percent $(n=3)$ presented with frank hematuria and flank pain, while the other $40 \%(n=2)$ had frank hematuria alone. The presence of hematuria was cited as the main reason for immediate ED attention. In all cases, urine dipstick was positive for blood. None of the patients had concomitant injuries.

All patients were investigated with triphasic CT scan as per established hospital protocols for the investigation of abdominal trauma. AAST-OIS grading for the 5 patients included 2 Grade III injuries and one of Grade I, II and IV (fig. 1).

All patients were admitted for clinical monitoring, with strict bed-rest and serial hematological and biochemistry checks. Mean hemoglobin on admission was $14.3 \mathrm{~g} / \mathrm{dl}$ (range 13.5-14.8 g/dl). Hemoglobin was monitored for an average of 2.5 days post admission (range 0-3 days). All patients were hemodynamically stable on presentation. All patients' hemoglobin remained stable from admission to discharge. None required blood transfusion. Mean creatinine on admission was $93.4 \mu \mathrm{mol} / \mathrm{l}$ (median $86 \mu \mathrm{mol} / \mathrm{l}$, range $82-119 \mu \mathrm{mol} / \mathrm{l}$ ). Patient five was noted to have an elevated creatinine $(119 \mu \mathrm{mol} / \mathrm{l})$ that normalized within 2 days after intravenous fluids. Creatinine remained stable in all other patients. Median length of stay for the patients during their acute presentation was 7 days (range 5-12 days).

Patient four underwent angiography in interventional radiology, to investigate a suspected vascular injury noted on CT scan. A small pseudo-aneurysm was noted on angiography, no procedure was required (fig. 2). 


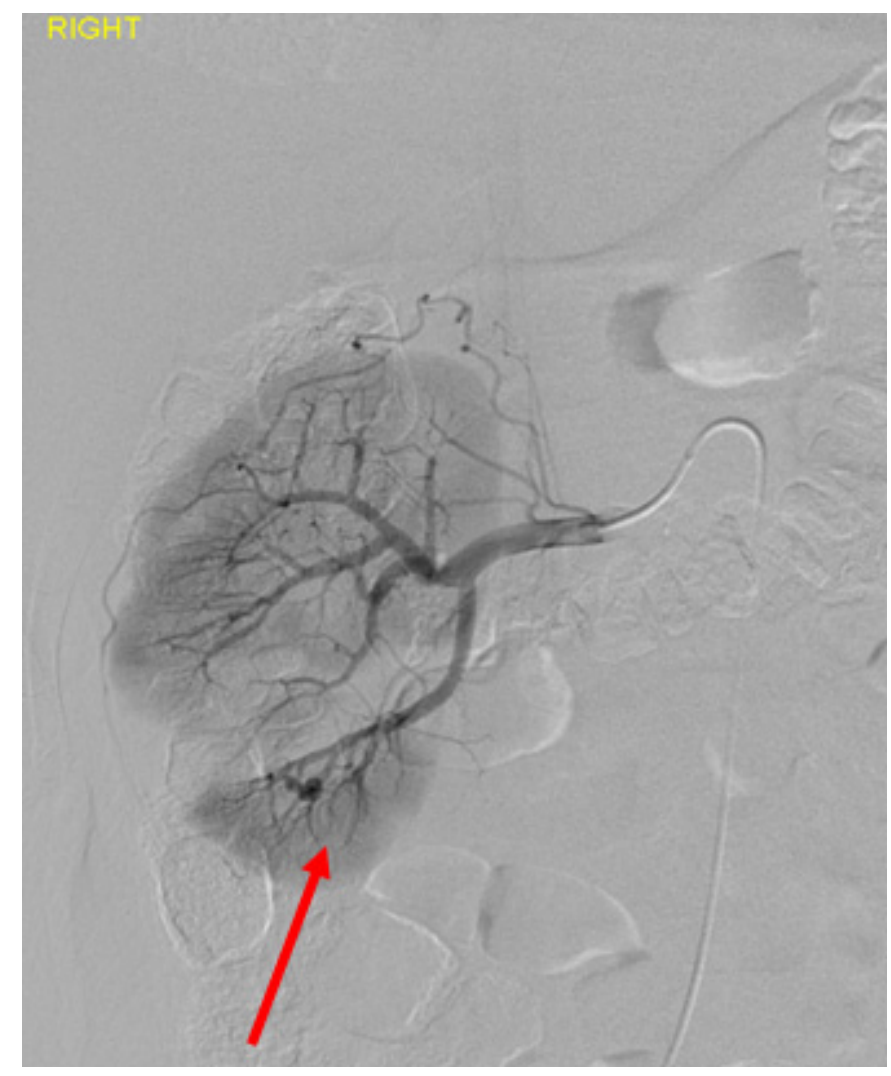

Fig. 2. Angiogram showing small pseudo-aneurysm on patient four.

All other patients were managed conservatively. Patient five underwent an elective left laparoscopic nephrectomy 8 week post injury after split renal function testing using Tc-99m diethylenetriamine pentaacetic acid observed a non-functioning kidney. The decision to proceed to nephrectomy was to minimize the high risk of re-bleeding from repeated abdominal rugby contact.

All other patients were followed-up in the urology outpatients department 6 weeks post injury with no acute issues. Three had recommenced rugby training prior to follow-up with urology on their own accord (table 1).

\section{Discussion}

The kidney is the most commonly injured genitourinary organ, occurring in 5\% of all trauma cases [1]. Though the main etiology for renal trauma is secondary to high velocity mechanisms including road traffic accidents and falls, there is an increasingly incidence of renal injuries secondary to sporting injuries. The presence of pre-existing renal abnormalities makes renal injury more likely following trauma [2], like with patient five in our study (though incidentally detected).

There are currently 26 classifications for renal injuries that have been reported in the literature over the past 60 years [7]. The most widely accepted classification is the AAST-OIS. Injuries are Graded I-IV in correlation with findings on abdominal CT [10]. The AAST score both aids management and confers a prognostic element [10]. Furthermore AAST scoring is the most important variable predicting the need for kidney repair or nephrectomy [4]. To date, there are no specific treatment guidelines pertaining to the short- and long-term management of sports-related trauma. EAU guidelines recommend imaging in all abdominal trauma patients with the presence of hematuria and shock or with major associated injuries. Furthermore, injury secondary to rapid deceleration warrants imaging to rule out ureteral avulsion or renal pedicle injury [11].

Traditionally, intravenous pyelography was the initial imaging modality in renal trauma cases [12]. However, it has been largely replaced by contrast CT scanning, and is only utilised in institutions without access to higher imaging modalities [13]. Contrast CT is the gold standard imaging modality. It provides good evaluation of inter abdominal and retroperitoneal structures, with excellent sensitivity of detecting concomitant injuries. CT has a negative predictive value of $99.8 \%$ [14].

The management of renal trauma can be broadly divided into 3 groups: conservative, radiological (angioembolisation) and surgical. In recent years conservative management has become the mainstay in the acute management of renal trauma. Many advocate admission with strict bed rest, clinical monitoring, serial examinations and hemoglobin checks until resolution of hematuria, as done in our cases. Most trauma patients are evaluated promptly, and therefore bloods are sent ideally within 1 hour of injury. Evidence of elevated creatinine at initial assessment is more reflective of pre-existing pathology.

Angiography and selective embolisation is utilised with success rates as high as $83 \%$ for management of Grade IV and V renal injuries with vascular complications [15]. Historically all Grade V injuries underwent surgical exploration, but this practice has become more controversial in contemporary times. Surgical exploration is now reserved for those patients presenting with significant renal trauma with associated hemodynamic instability [16]. Therefore, proceeding to surgery is taken on a clinical basis rather than solely on radiological find- 


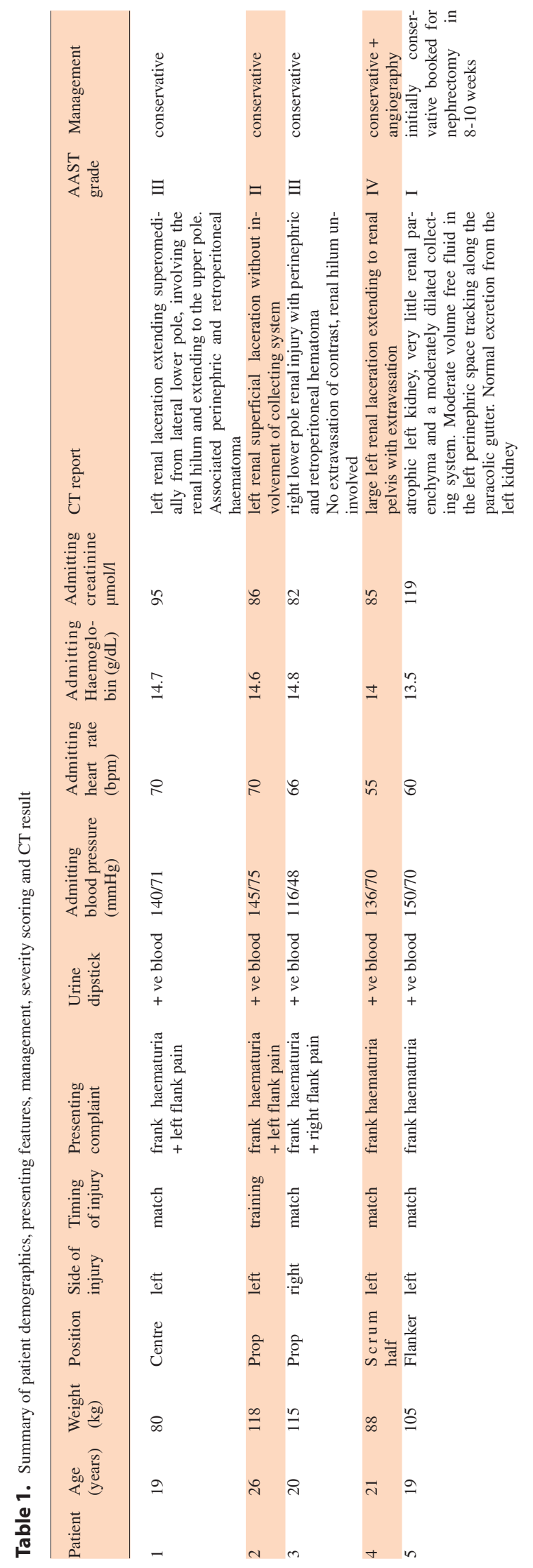

ings. The goal of surgery is to control hemorrhage and salvage the kidney, with reconstruction feasible in the majority of cases. Long-term studies have shown that conservative management has no impact on immediate or long-term morbidity or mortality rates [17]. The role of endoscopic surgical procedures with deployment of retrograde ureteral stents in cases where significant extravasation of urine is evident on CT should be considered to prevent urinoma formation. However, the treatment of each trauma case is unique and therefore should be considered on a case-by-case basis. Conservative treatment with antibiotics may suffice, however on occasions the placement of a percutaneous drain or ureteral stent may be required.

Despite the lack of benefit and strong evidence against repeat imaging, institutional protocols and "best" practices have a significant bearing on the role of repeated imaging. Some suggest re-imaging upon resolution of microscopic hematuria, others prior to discharge, while some repeat CT scans 6-10 weeks after initial injury [18]. EAU advises that Grade I-IV injuries do not necessitate repeat imaging unless there is an acute change in the patient's status (as is the practice in our institution) [19]. Furthermore the EAU recommends that longterm follow-up imaging should ultimately be decided on a case-by-case basis (Level C evidence) [19]. In the context of professional sport, physicians can face pressure to re-scan patients in an effort to shorten absence from sport. A survey of the American Medical Society of Sports Medicine physicians highlighted discrepancies between recommendations and clinical practice. Only $54 \%$ of members would formally advise athletes with a solitary kidney to re-participate in contact sports at high school or college level [20]. Evidence of renal injury whilst playing sport is sparse and anecdotal. A review of 4.4 million sport exposures found that kidney injury accounted for $0.076 \%$ of injuries recorded, with none requiring surgical management. Injuries to the brain, head, neck and spine are more prevalent [21]. Moreover, road traffic accidents alone are 2-10 times a more common cause of renal injury than sport [22], but physicians are unlikely to advise patients not to travel in motor vehicles. Ultimately patients/players must decide whether they want to return to sport, while aware of the risk.

Interestingly, an Irish study observed that field position correlated to risk of renal injury among rugby players [22]. There is a discernible difference in body habitus and weight depending on player position in rugby. Wing forward was the position most likely to give rise to injuries and prop was the least. The average weight of the 
current Irish rugby squad is $103.5 \mathrm{~kg}$, with a difference of $37 \mathrm{~kg}$ between the heaviest and lightest players, however it remains unclear whether body weight has any impact on risk and injury severity. The modern professional era of rugby has changed in recent years with players that are now bigger and faster therefore a significant higher risk of injury [23].

Timing of return to sporting activity is of significant concern to both amateur and professional athletes. The decision must be decided on an individual basis. However, prior to resuming sporting activity the potential complications and long-term sequelae should been discussed thoroughly. The lack of specific international guidelines and consensus, along with paucity of studies makes for difficult decision-making. Athletes, with counsel from appropriate physicians, ultimately must decide if and when to return to sport.

\section{Conclusion}

Rugby-related trauma though rare, has generated attention and awareness in recent times. It poses a conundrum for physicians and players due to the absence of clear guidelines and a paucity of scientific evidence. This paper serves to highlight this type of injury and the need for defined guidelines on role of imaging and international consensus on timing of return to contact sport, in both professional and amateur settings.

\section{References}

1 Santucci RA, Wessells H, Bartsch G, Descotes J, Heyns CF, McAninch JW, Nash P, Schmidlin F: Evaluation and management of renal injuries: consensus statement of the renal trauma subcommittee. BJU Int 2004; 93:937-954.

2 Lloyd GL, Slack S, McWilliams KL, Black A, Nicholson TM: Renal trauma from recreational accidents manifests different injury patterns than urban renal trauma. J Urol 2012;188:163-168.

3 Emanuel B, Weiss H, Golin P: Renal trauma in children. J Trauma 1977;17:275-278.

4 Santucci RA, Fisher MB: The literature increasingly supports expectant (conservative) management of renal trauma- a systematic review. J Trauma 2005;59:491-503.

5 Blankenship JC, Gavant ML, Cox CE, Chauhan RD, Gingrich JR: Importance of delayed imaging for blunt renal trauma. World J Surg 2001;25:1561-1564.

6 Alonso RC, Nacenta SB, Martinez PD, Guerrero AS, Fuentes CG: Kidney in danger: CT findings of blunt and penetrating renal trauma. Radiograpics 2009;29:2033-2053.

7 Djakovic N, Plas E, Martinez-Pineiro L, Lynch T, Mor Y, Santucci RA, Serafetinidis E, Turkeri LN, Hohenfellner M: Guidelines on Urological Trauma. European Association of Urology, 2008.
8 Sharma D, Butt N, Barrass B, Dawson C: Examining the impact of renal trauma guidelines on urologists in UK. Eur J Trauma Emerg Surg 2008;34:154-158.

9 Saint Vincent's University Hospital. Patient Information. Dublin, 2008.

10 Bernard JJ: Renal trauma: evaluation, management and return to play. Curr Sports Med Rep 2009;8:98-103.

11 Moore EE, Shackford SR, Pachter HL, McAninch JW, Browner BD, Champion HR, Flint LM, Gennarelli TA, Malangoni MA, Ramenofsky ML: Organ injury scaling: spleen, liver, and kidney. J Truama 1989;29: 1664-1666.

12 Heyns CF: Renal trauma: indications for imaging and surgical exploration. BJU Int 2004; 93:1165-1170.

13 Kawashima A, Sandler CM, Corl FM, West OC, Tamm EP, Fishman EK, Goldman SM: Imaging of renal trauma: a comprehensive review. Radiograhics 2001;21:557-574.

14 Bent C, Iyngkaran T, Power N, Matson M, Hajdinjak T, Buchholz N, Fotheringham T: Urological injuries following trauma. Clin Radiol 2008;63:1361-1371

15 Hom J: The risk of intra abdominal injuries in pediatric patients with stable blunt abdominal trauma and negative abdominal computed tomography. Acad Emerg Med 2010;17:469_ 475 .
16 Hotaling JM, Sorensen MD, Smith TG 3rd, Rivara FP, Wessells H, Voelzke BB: Analysis of diagnostic angiography and angioembolisation in the acute management of renal trauma using a national data set. J Urol 2011; 185:1316-1320.

17 Schmidlin FR, Rohner S, Hadaya K, Iselin CE, Vermeulen B, Khan H, Farshad M, Niederer P, Graber P: The conservative treatment of major kidney injuries. Ann Urol (Paris) 1997;31:246-252.

18 Walter KD: Radiographic evaluation of the patient with sport-related abdominal trauma Curr Sports Med Rep 2007;6:115-119.

19 Summerton DJ, Djakovic N, Kitrey ND, Kuehhas F, Lumen N, Serafetinidis E: Guidelines on urological trauma. European Association of Urology, 2013.

20 Anderson CR: Solitary kidney and sports participation. Arch Fam Med 1995;4:885-888.

21 Grinsell MM, Butz K, Gurka MJ, Gurka KK, Norwood V: Sport related kidney injury among high school athletes. Pediatrics 2012; 130:2011-2082.

22 Ryan JM, McQuillan R: A survey of rugby injuries attending an accident and emergency department. Ir Med J 1992;85:72-73.

23 Cheng HL, O'Connor H, Kay S, Cook R, Parker H, Orr R: Anthropometric characteristics of Australian junior representative rugby league players. J Sci Med Sport 2014;17: 546-551. 